\title{
The Dynamic Characteristics of Typhoon Vortex Spiral Waves and its Transportation: Diagnostic Analyses
}

\author{
Xu Xiangde Zhang Shengjun Chen Lianshou Wei Fengying \\ (Chinese Academy of Meteorological Sciences, Beijing 100081)
}

\section{Introduction}

The structure of typhoon spiral cloud band and its transportation characteristics are difficult theoretical problems in scientific field. Two main theories have been proposed to explain the physics of typhoon spiral bands (Abdullah 1966, Kurihara and Tuleya 1974, Kurihara 1976, Guinn and Schubert 1993, MacDomald 1968). But for absence of high-resolution information on typhoon structure, or in another word, for limitation of sounding techniques on inner-typhoon cloud structure, it is very difficult to further recognize the dynamic transportation characteristics of typhoon. The construction of related theoretical model always barges up against an insuppressible "bottleneck" problem. Recently, the satellite remote sensing technique has broken through this limitation at sea regions, especially that it can provide micro-phenomenon such as meso- and micro-scale structures of typhoon system, which would further disclose the transportation theory of above spiral waves.

\section{Data and calculation method}

Taking typhoon center as origin of moving-coordinate, we adopt statisticaldynamic method to study its vortex dynamic characteristics. We select Fred(1994), Winnie(1997), Dan(1999) as target typhoons, TBB and T106 reanalysis data as the data source. With aim to disclose the structure of real typhoon spiral wave and its transportation

Corresponding author address: Xu Xiangde, CAMS, 46 South Zhongguancun Street, 100081, Beijing, China, cep99@cams.cma.gov.cn features, correlation statistics, power spectrum method and nine-point filter analysis are used in this paper.

\section{Results}

1) The TBB significant correlation coefficient fields between typhoon eye wall cloud and its surrounding convective cloud show spiral wave band pattern and its transportation direction is in consistent with typhoon movement direction to some extent (Fig.1).
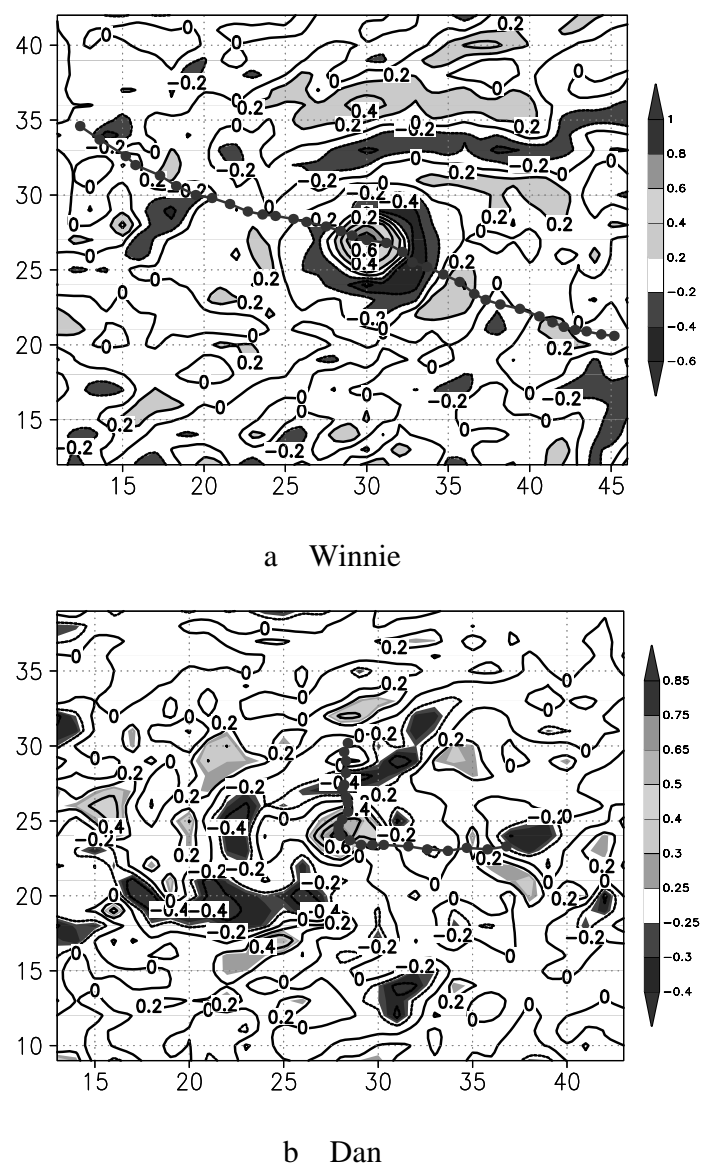

Figure 1 the correlation coefficient field between the disturbed $\mathrm{T}_{\mathrm{BB}}$ on the eye and around it in addition to the track of typhoon (shaded area has significance exceeding $90 \%$, dot-solid line is track of typhoon) 
2) The spiral waves in typhoon-effected regions show remarkable sub-synoptic and meso-scale perturbation feature, that is, the time scale of its transportation is similar to period of vortex Rossby wave or internal gravity wave with dominant period as about 6 or $24 \mathrm{~h}$. The point rainfall period-oscillation in typhoon-effected regions also displays above periodic feature. Furthermore, the significance of meso-scale perturbation in typhoon spiral band has close relation with typhoon vortex intensity.

3) At low level, the sub-synopitc and meso-scale convective cloud in spiral bands might lead to enhancement of typhoon perturbation through the spiral inflow. Following the outward transporting spiral waves these perturbations will trigger the development of surrounding convective cloud.. This kind of spiral wave transportation might have important effects on typhoon-CISK mechanism.

4) The structure model of typhoon spiral wave train further discloses that typhoon vortex has remarkable vortex Rossby wave feature, which reflects the dynamic effect of wave-stream interaction in typhoon vortex basic state (Fig.2).

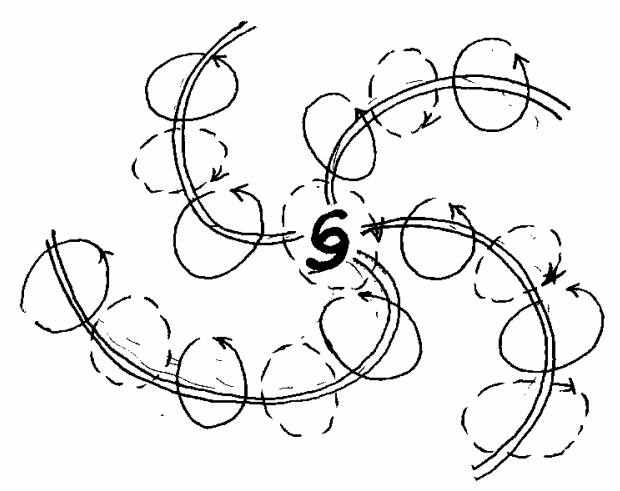

Figure2 the structure model of typhoon vortex spiral

wave

\section{References}

Abdullah, A. J., 1966: The spiral bands of a hurricane: a possible dynamic explanation.

\section{J. Atmos. Sci. 23, 367-375}

Kurihara, Y., 1976: On the development of spiral bands in tropical cyclone. J. Atmos. Sci., 33,940-958

Kurihara, Y., Tuleya, R. E., 1974: Structure of tropical cyclone developed in a three dimensional numerical simulation model. J. Atmos. Sci., 31,893-199

Mac Donald, N. J., 1968: The evidence for the existence of Rossby-like waves in the hurricane vortex. Tellus, 20, 138-150

Guinn, T. A., W. H. Schubert, 1993: Hurricane spiral rainband. J. Atmos. Sci., 50,3380-3403 